\title{
A phase-I trial of pre-operative, margin intensive, stereotactic body radiation therapy for pancreatic cancer: the 'SPARC' trial protocol
}

Daniel L. P. Holyoake', Elizabeth Ward², Derek Grose ${ }^{3}$, David Mclntosh³, David Sebag-Montefiore ${ }^{4,5}$, Ganesh Radhakrishna ${ }^{5}$, Neel Patel ${ }^{6}$, Michael Silva ${ }^{6}$, Somnath Mukherjee ${ }^{1,6}$, Victoria Y. Strauss ${ }^{7}$, Lang'o Odondi ${ }^{7}$, Emmanouil Fokas ${ }^{1}$, Alan Melcher ${ }^{8}$ and Maria A. Hawkins ${ }^{1 *}$

\begin{abstract}
Background: Standard therapy for borderline-resectable pancreatic cancer in the UK is surgery with adjuvant chemotherapy, but rates of resection with clear margins are unsatisfactory and overall survival remains poor. Meta-analysis of single-arm studies shows the potential of neo-adjuvant chemo-radiotherapy but the relative radioresistance of pancreatic cancer means the efficacy of conventional dose schedules is limited. Stereotactic radiotherapy achieves sufficient accuracy and precision to enable pre-operative margin-intensive dose escalation with the goal of increasing rates of clear resection margins and local disease control.

Methods/Design: SPARC is a "rolling-six" design single-arm study to establish the maximum tolerated dose for margin-intensive stereotactic radiotherapy before resection of pancreatic cancer at high risk of positive resection margins. Eligible patients will have histologically or cytologically proven pancreatic cancer defined as borderline-resectable per National Comprehensive Cancer Network criteria or operable tumour in contact with vessels increasing the risk of positive margin. Up to 24 patients will be recruited from up to 5 treating centres and a 'rolling-six' design is utilised to minimise delays and facilitate ongoing recruitment during doseescalation. Radiotherapy will be delivered in 5 daily fractions and surgery, if appropriate, will take place 5-6 weeks after radiotherapy. The margin-intense radiotherapy concept includes a systematic method to define the target volume for a simultaneous integrated boost in the region of tumour-vessel infiltration, and up to 4 radiotherapy dose levels will be investigated. Maximum tolerated dose is defined as the highest dose at which no more than 1 of 6 patients or 0 of 3 patients experience a dose limiting toxicity. Secondary endpoints include resection rate, resection margin status, response rate, overall survival and progression free survival at 12 and 24 months. Translational work will involve exploratory analyses of the cytological and humoral immunological responses to stereotactic radiotherapy in pancreatic cancer. Radiotherapy quality assurance of target definition and radiotherapy planning is enforced with pre-trial test cases and on-trial review. Recruitment began in April 2015.

(Continued on next page)
\end{abstract}

\footnotetext{
* Correspondence: maria.hawkins@oncology.ox.ac.uk

${ }^{1}$ CRUKMMRC Oxford Institute for Radiation Oncology, Department of

Oncology, University of Oxford, Old Road Campus Research Building,

Roosevelt Drive, Oxford OX3 7DQ, UK

Full list of author information is available at the end of the article
} 
(Continued from previous page)

Discussion: This prospective multi-centre study aims to establish the maximum tolerated dose of pre-operative margin-intensified stereotactic radiotherapy in pancreatic cancer at high risk of positive resection margins with a view to subsequent definitive comparison with other neoadjuvant treatment options.

Trial registration: ISRCTN14138956. Funded by CRUK

Keywords: Pancreatic cancer, Borderline-resectable, Stereotactic body radiation therapy (SBRT)

\section{Background}

Pancreatic cancer is responsible for only $3 \%$ of cancer diagnoses but $5 \%$ of cancer deaths, making it the fifth leading cause of cancer death in the UK (9408 cases in 2013 and 8817 deaths in 2014) [1]. Radical surgical resection represents the only chance of long-term disease control, but was possible for just $8 \%$ of UK patients diagnosed in 2006-2010 [2]. In Europe standard practice for resectable disease is surgery followed by chemotherapy, but for these patients median survival is only 24 months and $50 \%$ of patients will suffer local recurrence [3] suggesting that further improvement in multimodal therapy is required.

The failure to consistently achieve microscopic surgical clearance contributes to the high rates of disease relapse: alongside tumour size, grade and lymph node metastases, resection margin involvement has been repeatedly shown to predict long-term survival [4-6]. Accurate margin assessment requires comprehensive examination of the surgical specimen and rates of margin involvement are higher when a standardised protocol is used [7]. Despite centralization of services and improvement in diagnostic radiology, positive margins were reported in $>35 \%$ of patients in the largest multi-national adjuvant trial in pancreatic cancer involving over 1000 patients (ESPAC 3), which again reinforced that patients with positive margins have poor outcome (hazard ratio 1.35, $95 \%$ confidence interval 1.17-1.56, $p<0.001$ ) [8].

A small proportion of patients present with pancreatic tumours that can be classified as "borderline-resectable", defined by a limited extent of vascular invasion. For these patients resection is possible but is likely to require vascular reconstruction and there is an especially high risk of positive resection margins, reported to be $62.9 \%$ in the UK [9]. The concept of Borderline Resectable Pancreatic Cancer (BRPC) was initially described in 2001 by Mehta et al., describing 15 cases initially assessed as "marginally resectable" but where resectability was improved with preoperative chemoradiotherapy [10]. The definition of BRPC was refined by an AHPBA/SSO/SSAT consensus statement [11, 12], now adopted by the NCCN [13]:-

- Venous involvement of the SMV or PV with distortion or narrowing of the vein or occlusion of the vein with suitable vessel proximal and distal, allowing safe resection and reconstruction

- Gastro-duodenal artery encasement up to hepatic artery with either short segment encasement or direct abutment of the hepatic artery, without extension to the celiac axis

- Tumour abutment of the SMA not to exceed greater than $180^{\circ}$ of the circumference of the vessel wall.

An alternative definition of BRPC by the MDACC has not been as widely recognised [14]. A multidisciplinary team approach is needed to assess the likelihood of attaining negative margins and this should remain the key consideration in determining if a patient is a potential candidate for resection $[14,15]$.

The management of BPRC is controversial, and there is increased interest in pre-operative treatment strategies that may improve outcomes [16]. Theoretical advantages include a greater chance of delivering full dose treatment, while drawbacks include possible overtreatment of resectable disease, the need for a biopsy and stent to relieve obstruction while awaiting surgery. Many patients presenting with localised disease develop frank metastases shortly after diagnosis and if this occurs during induction therapy these patients can be spared inappropriate and futile surgery [14].

Pre-operative (chemo) radiotherapy aims to treat the tumour in situ to achieve tumour regression and facilitate curative resection. This approach has become standard of care for some tumour sites, having been shown to reduce local recurrence in rectal cancer [17], and increase overall survival in oesophageal cancer [18], but is not established in pancreatic cancer due to limited effectiveness of standard dose schedules, despite potential advantages over postoperative treatment [19]. A complete tumour response is only seen in around $5 \%$ of patients [20], though a larger proportion of patients (35\%) benefit from tumour down-staging to achieve resectability [21] and patients who successfully complete neoadjuvant chemoradiotherapy and go on to have resection tend to have high rates of clear resection margins $[22,23]$ and reduced rates of local recurrence [24,25].

Stereotactic body radiation therapy (SBRT) combines rigorous immobilisation and image-guidance with hypofractionation, to reduce radiotherapy planning margins 
and treat small targeted volumes with greater accuracy and precision, supporting safe delivery of much higher biologically effective doses. It has been shown to be a highly-effective local treatment option in unresectable pancreatic cancer with high rates of local control achieved using treatment delivered in a single exposure [26-29], and when treatment was delivered in 3-5 fractions lower rates of toxicity were seen without compromising disease control [30-35].

The specific goal in treating BRPC with pre-operative radiotherapy will be improve the chances of achieving clear resection margins, reduce risk of local recurrence and achieve chances of long-term survival similar to those for patients with initially resectable tumours. Neoadjuvant treatment has been shown to be deliverable and effective in BRPC, such as in a series of 160 patients described by Katz et al., among whom $78 \%$ completed preoperative therapy and restaging, and $41 \%$ underwent pancreatectomy, with a $94 \%$ rate of clear margins [36].

The premise of the SPARC trial is a Margin-Intense radiotherapy concept where the whole tumour is treated to a minimum dose while a simultaneous integrated boost (SIB) is used to deliver additional dose to the margin of tumour around structures such as the superior mesenteric artery (SMA), SMV or portal vein and retroperitoneal margin. This approach complements surgery as these are the most difficult margins to resect and are at highest risk of positive surgical margins. Prospective dose-escalation aims to establish the maximum tolerated dose for this treatment paradigm for subsequent definitive investigation of efficacy. Once the maximum tolerated dose (MTD) is established this will provide a platform to integrate with optimal systemic treatment.

\section{Methods/Design Study design}

The SPARC trial is a phase I dose-escalation study using the rolling six design [37] to establish MTD of SBRT delivered in the pre-operative setting for borderline resectable pancreatic cancers. This method minimises delays in the dose escalation phase [37]. Patients will complete SBRT in approximately one week and surgery will take place 5-6 weeks after radiotherapy. Patients will be on the study for 36 weeks from registration to end of treatment visit followed by standard care for two years post SBRT day one for the last patient, or when all participants have died.

The trial opened in April 2015 and will recruit up to 24 patients from five UK centres (Oxford, Glasgow, Leeds, Newcastle and Nottingham). The SPARC trial is conducted in accordance with the Helsinki Declaration (1996) and the regulatory requirements for clinical trials of an investigational medicinal product under the European Union Clinical Trials Directive. The trial is approved by the National Research Ethics Service Committee South Central - Oxford B (REC reference: $15 / \mathrm{SC} / 0059)$ and is sponsored by the University of Oxford, with funding from Cancer Research UK. Data submission for the SPARC trial is via electronic submission into the online database system OpenClinica by site staff. The Oncology Clinical Trial Office (OCTO) coordinates and manages the trial while the Centre for Statistics in Medicine (University of Oxford) will perform all statistical analysis.

\section{Participants}

The inclusion and exclusion criteria for the SPARC trial are summarised in Table 1.

\section{Interventions}

At registration patients are assigned to the appropriate SBRT dose level (Table 2) and will receive 5 fractions of SBRT over 5-8 days. The dose schedules selected have been designed to achieve approximately equivalent dose to the tumour to that used in conventionally fractionated radical radiotherapy (50-66Gy in 2Gy fractions) while the dose selected for the 'at risk volume' (area at risk of positive margin) is higher, to achieve increased likelihood of ablation of tumour cells. A maximum of 4 dose levels (including one de-escalated dose level) are expected to be needed to establish the MTD.

Dose level assignment will be based on the number of dose limiting toxicities (DLTs) observed, and the number of patients have no DLTs yet, but are still in the DLT assessment period as defined in the outcome measure below (i.e., toxicity data pending/evaluable not yet). In this trial design, the first two patients are treated at level 1. The third patient will be treated at level -1 if two DLTs have been observed. If at least one patients enrolled is not evaluable yet and less than 2 DLTs have been observed, this third patient will be entered at the same dose level, Level 1. For the fourth patient, if toxicity data is pending for at least one of the patients enrolled, the fourth patient will be allocated to the same dose level. But, if there are two or more DLTs when the fourth patient enters the study, the dose level will be deescalated. The dose level will be escalated if there is no DLT after the first three patients finish the DLT assessment period. This process will be repeated for patients five and six. After the sixth patient is enrolled to a dose cohort, accrual will be only temporarily suspended until toxicity data is fully available for these six patients and/ or a decision from the Trial Management Group (TMG) is made. Recruitment will stop once the MTD is identified. 
Table 1 SPARC Inclusion/Exclusion criteria

\begin{tabular}{|c|c|}
\hline clusion criteria & riteria \\
\hline $\begin{array}{l}\text { 1. Borderline resectable localised tumour of the pancreatic head/uncinate } \\
\text { process/body as per NCCN Guidelines (tumours of the tail of pancreas } \\
\text { are not eligible for inclusion) or operable tumour in contact with vessels } \\
\text { increasing the risk of positive margin as defined by CT }+/- \text { MRI }+/- \text { PET } \\
\text { criteria within } 28+/-7 \text { days prior to trial entry, de novo or following } \\
\text { systemic treatment. } \\
\text { 2. Histologically proven pancreatic ductal adenocarcinoma or cytological } \\
\text { proven pancreatic malignancy } \\
\text { 3. Able to undergo biliary drainage using a stent } \\
\text { 4. Deemed fit and suitable for surgical resection. } \\
\text { 5. No overt metastases or uncertain status with investigations suspicious } \\
\text { of possible metastatic disease (e.g., small equivocal pulmonary nodule(s)). } \\
\text { 6. Male or female, Age } \geq 16 \text { years } \\
\text { 7. Life expectancy of at least } 6 \text { months } \\
\text { 8. ECOG performance status } 0-1 \\
\text { 9. The patient is willing and able to comply with the protocol for the duration } \\
\text { of the study, and scheduled follow-up visits and examinations } \\
\text { 10. Written (signed and dated) informed consent and be capable of } \\
\text { co-operating with protocol } \\
\text { 11. Haematological and biochemical indices within specified ranges. }\end{array}$ & $\begin{array}{l}\text { 1. Definitive metastatic disease or local disease that cannot be } \\
\text { encompassed in the SBRT field. } \\
\text { 2. History of previous or concurrent malignancy diagnoses for which } \\
\text { the expected prognosis is likely to be worse than that of the current } \\
\text { diagnosis of pancreatic cancer (excludes for example: e.g., localised } \\
\text { prostate cancer, early colorectal cancer, early breast cancer, } \\
\text { curatively-treated basal cell carcinoma of skin, carcinoma in situ of } \\
\text { cervix; curatively treated cancer of other sites who are recurrence } \\
\text { free for }>3 \text { years). } \\
\text { 3. Serious medical or psychological condition precluding trial } \\
\text { intervention. } \\
\text { 4. Previous upper abdominal or right chest wall radiotherapy where } \\
\text { 30\% of the liver has received }>15 G y \text {. } \\
\text { 5. Pregnancy: Pregnant or breast-feeding women are ineligible. } \\
\text { Women of childbearing potential must use effective methods of } \\
\text { contraception. } \\
\text { 6. Any other psychological, social or medical condition, physical } \\
\text { examination finding or laboratory abnormality that the Investigator } \\
\text { considers makes the patient a poor trial candidate or could interfere } \\
\text { with protocol compliance or the interpretation of the trial results. }\end{array}$ \\
\hline
\end{tabular}

\section{Outcome measures and definitions}

The primary endpoint of the SPARC study is MTD, defined as the highest level of SBRT at which no more than 1 of 6 patients or 0 of 3 patients experiences a DLT in the period from starting SBRT to one month postsurgery or one month after the evaluation in week 3 post-SBRT if no surgery takes place. DLT is defined as these possible adverse events (AEs) (according to CTCAE v4.03), seen in the DLT assessment period:

1. $\geq$ Grade 3 upper gastro-intestinal (GI) bleeding

2. $\geq$ Grade 4 nausea/vomiting uncontrolled after $48 \mathrm{~h}$ of standard treatment

3. $\geq$ Grade 4 pancreatitis not stent related

4. Interruption of SBRT $>1$ week due to SBRT-related AEs

5. $\geq$ Grade 4 vascular events: SMV thrombosis, bowel ischaemia due to SMA arteritis/stenosis, friable vessels at surgery

6. Other AEs that the TMG agrees to be dose limiting and possibly related to SBRT such as $\geq$ grade 3 gastrointestinal fistula $>30$ days after surgery

Secondary outcomes include resection rate, resection margin status rates, rate of pathological complete response, late SBRT toxicity, efficacy and long term safety. Resection rate is defined as the proportion of patients with radical oncological resection of pancreatic cancer over the total enrolled patients. Resection margin status (R0/R1/R2) is defined according to the standards of the Royal College of Pathologists, and response rate is defined as the rate of patients with pathological complete response over the total enrolled patients. Late toxicity is defined as any GI AE/other $\mathrm{AE}>$ grade 2 occurring between 1 month and 6 month post-surgery. Additional secondary outcomes include efficacy and long term safety as indicated by progression free survival (PFS) and overall survival (OS). PFS is defined as the time from date of first SBRT dose to the first date of progression or death for any cause, or is censored at the date of last known follow-up date if they are not observed to die during the course of the trial. OS is defined as the time from date of first SBRT dose to the date of death for any cause or is censored at the last known follow-up date. Exploratory outcomes include relationships between imaging and pathology (treatment response and resection margin status) and changes in markers of the innate and adaptive immune response before and during SBRT (cytological assessments and levels of interferon-related RNA).

Table 2 Radiotherapy dose escalation levels

\begin{tabular}{|c|c|c|c|c|c|c|c|c|}
\hline \multirow{2}{*}{$\begin{array}{l}\text { Radiotherapy } \\
\text { dose level }\end{array}$} & \multicolumn{4}{|l|}{ Tumour (PTV) } & \multicolumn{4}{|c|}{ Area at risk of R1 (PTV_R) } \\
\hline & Dose/\# [Gy] & Total dose [Gy] & ${ }^{*} B E D\left[G y_{10}\right]$ & $\mathrm{EQD}_{2}[\mathrm{~Gy}]$ & Dose/\# [Gy] & Total dose [Gy] & *BED $\left[\mathrm{Gy}_{10}\right]$ & $\mathrm{EQD}_{2}[\mathrm{~Gy}]$ \\
\hline Level -1 & 6 & 30 & 50 & 40 & 8 & 40 & 72 & 60 \\
\hline Level 1 & 6 & 30 & 50 & 40 & 9 & 45 & 88 & 71.5 \\
\hline Level 2 & 6.5 & 32.5 & 56 & 45 & 9.5 & 47.5 & 92 & 77.2 \\
\hline Level 3 & 7 & 35 & 62 & 50 & 10 & 50 & 100 & 83.3 \\
\hline
\end{tabular}

*BED [Gy $\left.{ }_{10}\right]$ biologically effective dose for acute reacting tissues $(\alpha / \beta=10), E Q D_{2}$ equivalent dose in 2 -Gy fractions 


\section{Sample size considerations}

Patient numbers will be determined by the primary endpoint of establishing the MTD, which will require a maximum of 24 evaluable patients. An evaluable patient for primary endpoint analysis is defined as one who has received at least one fraction of SBRT and experiences a DLT, or a patient who has received at least four fractions of SBRT and is assessed one month post-surgery (or assessed at similar time for DLT if no surgery). Patients not evaluable for primary endpoint will be replaced, but a patient who does not become evaluable for the primary endpoint may continue to be followed up for surgical outcome, PFS and OS as appropriate, unless consent is withdrawn.

\section{Statistical analysis}

All analyses will follow a statistical analysis plan written in accordance with current standard operating procedures. Baseline characteristics will be summarised (number/frequency) for all enrolled patients. Patients who died or withdrew before treatment started or fail to complete the required safety observations will be described separately. Primary MTD analysis will concern the frequency of patients with no DLT according to dose-escalation of SBRT. Descriptive statistics will summarise the DLT's and safety variables with patients grouped according to dose level received. Secondary outcomes (resection rate, resection margin status and response rate) associated with SBRT will be summarised by descriptive statistics along with numbers and percentages. Late toxicity will be summarised by descriptive statistics for all patients who received at least one dose of SBRT treatment. Physical exam, haematology and biochemistry data will be summarised across time. Both PFS and OS estimates at one and two years and $90 \% \mathrm{CI}$ will be provided together with median estimates. A Kaplan-Meier curve will be plotted with $90 \% \mathrm{CI}$. A trial summary will record patient recruitment and trial decision-making.

\section{Monitoring committees}

The Radiotherapy and Imaging Trials Oversight Committee (RIOC) is the independent committee for SPARC covering both data management committee and oversight responsibilities as delegated by the Sponsor and will meet at least once annually to review and ratify substantial decisions.

\section{Discussion}

This study builds on the current evidence base in SBRT for pancreatic cancer, which has so far been largely used in the locally advanced setting with promising results and aims to take it a step further by prospectively evaluating the safety and benefit of pre-operative marginintense SBRT.
A five-fraction schedule aims to optimise the balance of short duration and tumouricidal biologically-effective dose to the tumour with acceptable risk of late normal tissue injury. Potential clinical benefits of a significantly hypofractionated radiotherapy regimen compared with a protracted conventional 6-week course of chemoradiotherapy include a shorter enforced delay to definitive surgery or alternatively more straightforward integration with systemic therapy regimens.

The concept of margin-intensive therapy aims to further improve local control without increasing toxicity, as the region of integrated boost tends not to overlap with the major dose-limiting organ at risk (duodenum) helping to make safe dose-escalation to this sub-volume possible. Normal-tissue dose-volume constraints retain priority but where there is overlap, only the dose to the overlapping region is constrained as necessary, enabling the non-overlapping portion of the target volume to receive the prescribed dose (see Fig. 1).

The optimal pre-operative therapy in pancreatic cancer has not been identified, and could involve chemotherapy, radiotherapy or both. The national multicentre study ESPAC-5 F is currently open to recruitment in the UK with the primary aim to establish the feasibility of recruiting patients with BRPC to be randomised between two pre-operative chemotherapy regimens (GEMCAP and FOLFIRINOX), pre-operative CRT and standard of care (surgery followed by adjuvant chemotherapy) (38), therapy options which would then require definitive comparative evaluation in a subsequent larger study.

Two single-institution retrospective reviews report outcomes for the use of SBRT in patients with BRPC, both of which show high rates of subsequent successful surgical resection with clear margins [38, 39]. Rajagopalan et al. [38] report on 12 resected patients, of which 7 were deemed BRPC using.

MDACC criteria. Most patients received Gemcitabinebased systemic chemotherapy. The SBRT consisted of 24-36 Gy in 3 fractions, surgery took place at a median 3.3 months (range $1.5-6.6$ months) and $11 / 12$ patients had an R0 resection. Chuong et al. [39] treated 73 patients (of which 57 were deemed BRPC using NCCN criteria) with systemic Gemcitabine-based chemotherapy and 5 -fraction SBRT. Median dose prescribed was 35 Gy to the tumour margin and no less than 25 Gy to the entire pancreatic tumour. 44/57 BRPC cases underwent exploratory resection and $31 / 32$ resected cases had an R0 resection. No acute toxicity > grade 3 occurred and $5.3 \%$ (4 patients with locally advanced inoperable disease) experienced late grade 3 toxicity ( 3 GI bleeding and 1 anorexia). With a median FU of 10.5 months (2.225.9 months) the median OS was 16.4 months in all BRPC patients, with median OS of 19.3 months in resected patients. Disappointing overall survival rates in 


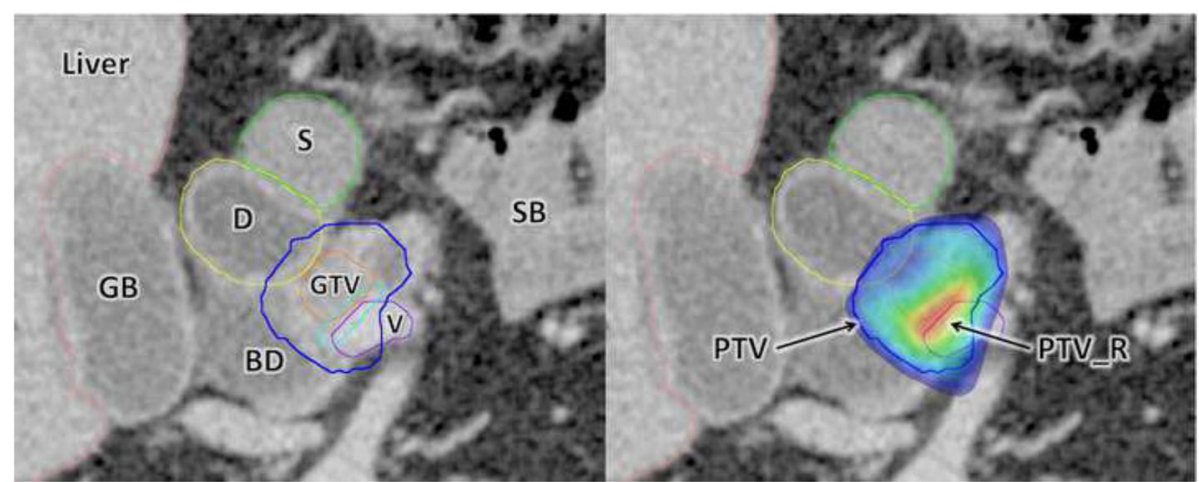

Fig. 1 Axial contrast-enhanced CT of patient with borderline-resectable pancreatic cancer demonstrating SPARC radiotherapy planning. Left-hand image - delineated structures: (clockwise from left) $G B=$ gall-bladder, $D=$ duodenum, $S=$ stomach, $S B=$ small-bowel, $V=$ vessel in contact with tumour, GTV = Gross Tumour Volume, BD = bile duct. Right-hand image - radiotherapy plan dose colourwash demonstrating dose levels delivered to PTV_R (light blue contour), PTV (dark blue) and PTV overlapping with duodenum

published series (median overall survival 5-20 months) are attributed to variable provision of optimal systemic treatment.

Evidence is growing for the importance of Radiation Therapy Quality Assurance (RTQA) for clinical trial interpretation as well as patient outcomes [40]. The SPARC trial incorporates a comprehensive prospective RTQA programme with a detailed radiotherapy protocol and atlas that was supported by a pre-trial workshop for collaborating clinicians, both of which have been shown to reduce variation in target volume definition [41-43]. A pre-trial test case of contouring and planning must be completed satisfactorily by recruiting centres and RTQA will continue with on-trial peer review prior to treatment supported by web-conferencing facilities.

The trial originally opened with eligibility criteria that excluded patients with suspicion of metastatic disease, those who may not be fit for surgical resection, and those who had received induction chemotherapy, which has become more common practice since the study was initially designed. Many patients presenting to our centre with BRPC were rendered ineligible for these reasons, and during the first year that the trial was open only one patient was successfully recruited. The primary endpoint of the study is the assessment of the safety and tolerability of the intervention, and therefore the patient eligibility inclusion and exclusion criteria for the study were revised to the current version published here, with the aim of improving patient recruitment.

\section{Exploratory immunological investigations}

Preclinical evidence suggests radiotherapy generates an immunological response, which can contribute to the efficacy of treatment. Direct damage and local inflammation increases antigen presentation to stimulate adaptive immune responses, and radiotherapy delivered in larger fractions has been shown to particularly enhance this effect [28]. In the SPARC study a panel of immunological tests to examine innate and adaptive immune responses to pancreatic SBRT. These preliminary data will be explored for their utility as predictive bio-markers in future studies.

\section{Conclusion}

The non-interventional phase of follow-up after the last patient last visit at the end of study will permit an estimate of the efficacy of pre-operative SBRT on overall survival and progression free survival to be observed. Once the MTD has been defined in this phase I trial, a phase II study will definitively evaluate efficacy endpoints. If positive, subsequent randomised phase III investigations will be required to assess superiority of this treatment strategy alone or integrated with pre-operative systemic treatment over surgery alone for this patient group.

\section{Abbreviations}

AHPBA: American Hepato-Pancreato-Biliary Association; ALP: Alkaline phosphatase; AST: Aspartate transaminase; BED: Biologically effective dose; BRPC: Borderline resectable pancreatic cancer; Cl: Confidence interval;

CRT: Chemo-radiotherapy; CRUK: Cancer Research United Kingdom; CSM: University of Oxford Centre for Statistics in Medicine; CT: Computed tomography; CTAAC: Cancer Research UK Clinical Trials Award and Advisory Committee; DLT: Dose-limiting toxicity; ESPAC: European study group for pancreatic cancer; $\mathrm{EQD}_{2}$ : Equivalent dose in 2-Gy fractions;

FOLFIRINOX: Folinic acid-5-fluourouracil-irinotecan-oxaliplatin; FU: Follow-up; GEMCAP: Gemcitabine-capecitabine; HR: Hazard ratio; MDACC: MD Anderson Cancer Centre; MDT: Multi-disciplinary team; MRI: Magnetic resonance imaging; MTD: Maximum tolerated dose; NCCN: National comprehensive cancer network; OCTO: Oncology clinical trials office; OCTRU: Oxford clinical trials research unit; OIRO: CRUK/MRC Oxford Institute for Radiation Oncology; OS: Overall survival; PFS: Progression-free survival; PV: Portal vein; REC: Research ethics committee; RIOC: Radiotherapy and imaging trials oversight committee; RTQA: Radiation therapy quality assurance; SBRT: Stereotactic body radiation therapy; SMA: Superior mesenteric artery; SMV: Superior mesenteric vein; SSAT: Society for surgery of the alimentary tract; SSO: Society of surgical oncology 
Trial Management Group:

Prof David Sebag-Montefiore, Dr Ganesh Radhakrishna, Mr Andrew Smith (Leeds Teaching Hospital NHS Trust).

Dr Derek Grose, Dr David McIntosh (Beatson Cancer Centre, Glasgow). Dr Somnath Mukherjee, Dr Neel Patel, Mr Michael Silva, Mr Maxwell Robinson (Oxford University Hospitals NHS Trust, Oxford).

Dr Victoria Strauss, Dr Lang'o Odondi (Centre for Statistical Medicine, Oxford). Translational research team: Dr Emmanouil Fokas (Oxford Institute for Radiation Oncology, Oxford), Professor Alan Melcher (The Institute of Cancer Research, London).

\section{Funding}

The trial is funded by Cancer Research UK (grant number C43735/A18787). DLPH is funded by a CRUK Nuffield Clinical Research Fellowship, grant code C5255. MAH is funded by the MRC (Medical Research Council), grant code MC_PC_12001/2.

\section{Availability of data and materials}

Additional data and materials may be requested from Professor Maria Hawkins.

\section{Authors' contributions}

DLPH drafted the manuscript and participated in trial design and coordination. VS \& LO developed the trial design and helped to draft the manuscript. MAH conceived the study, participated in its design and coordination and helped to draft the manuscript. All authors were involved in the trial design and have read and approved the final manuscript.

\section{Competing interests}

The authors declare that they have no competing interests.

\section{Consent to publish}

Not applicable.

\section{Ethics approval and consent to participate}

The trial is approved by the National Research Ethics Service Committee South Central - Oxford B (REC reference: 15/SC/0059). Signed informed consent of all participants is required.

\section{Author details}

'CRUK/MRC Oxford Institute for Radiation Oncology, Department of Oncology, University of Oxford, Old Road Campus Research Building, Roosevelt Drive, Oxford OX3 7DQ, UK. ${ }^{2}$ Oncology Clinical Trials Office, Department of Oncology, University of Oxford, Old Road Campus Research Building, Roosevelt Drive, Oxford OX3 7DQ, UK. ${ }^{3}$ The Beatson West of Scotland Cancer Centre, 1053 Great Western Rd, Glasgow G12 OYN, UK. ${ }^{4}$ The University of Leeds, Cancer Research UK Leeds Centre,14 Leeds Institute of Cancer and Pathology, Cancer Genetics Building, St James's University Hospital, 15 Beckett Street, Leeds, West Yorkshire LS9 7TF, UK. ${ }^{5}$ Leeds Cancer Centre, Leeds Teaching Hospitals NHS Trust, Bexley Wing, Beckett Street, Leeds LS9 7TF, UK. ${ }^{6}$ Oxford University Hospitals NHS Foundation Trust, Old Road, Headington, Oxford OX3 7LE, UK. ${ }^{7}$ Centre for Statistics in Medicine, Nuffield Department of Orthopaedics, Rheumatology and Musculoskeletal Sciences, University of Oxford, Botnar Research Centre, Windmill Road, Oxford OX3 7LD, UK. ${ }^{8}$ The Institute of Cancer Research, Chester Beatty Laboratories, 237, Fulham Rd, London SW3 6JB, UK.

Received: 8 August 2016 Accepted: 17 August 2016 Published online: 13 September 2016

\section{References}

1. Pancreatic cancer statistics. [http://www.cancerresearchuk.org/healthprofessional/cancer-statistics/statistics-by-cancer-type/pancreatic-cancer]. Accessed 9 Sept 2016

2. National Cancer Intelligence Network and Cancer Research UK. Major resections by cancer site, in England; 2006-2010. London: Public Health England; 2015. http://www.ncin.org.uk/view?rid=2972. Accessed 9 Sept 2016.

3. Oettle H, Neuhaus P, Hochhaus A, Hartmann JT, Gellert K, Ridwelski K, et al. Adjuvant chemotherapy with gemcitabine and long-term outcomes among patients with resected pancreatic cancer: the CONKO-001 randomized trial. JAMA. 2013;310(14):1473-81.
4. Sohn TA, Yeo CJ, Cameron JL, Koniaris L, Kaushal S, Abrams RA, et al. Resected adenocarcinoma of the pancreas-616 patients: results, outcomes, and prognostic indicators. J Gastrointest Surg. 2000;4(6):567-79.

5. Howard TJ, Krug JE, Yu J, Zyromski NJ, Schmidt CM, Jacobson LE, et al. A margin-negative $\mathrm{RO}$ resection accomplished with minimal postoperative complications is the surgeon's contribution to long-term survival in pancreatic cancer. J Gastrointest Surg. 2006;10(10):1338-45. discussion 1345-1336.

6. Ghaneh P, Costello E, Neoptolemos JP. Biology and management of pancreatic cancer. Gut. 2007;56(8):1134-52.

7. Verbeke CS, Menon KV. Redefining resection margin status in pancreatic cancer. HPB (Oxford). 2009:11(4):282-9.

8. Neoptolemos JP, Stocken DD, Bassi C, Ghaneh P, Cunningham D, Goldstein $D$, et al. Adjuvant chemotherapy with fluorouracil plus folinic acid vs gemcitabine following pancreatic cancer resection: A randomized controlled trial. JAMA. 2010;304(10):1073-81.

9. Ravikumar R, Sabin C, Abu Hilal M, Bramhall S, White S, Wigmore S, et al. Portal vein resection in borderline resectable pancreatic cancer: a United Kingdom multicenter study. J Am Coll Surg. 2014;218(3):401-11.

10. Mehta VK, Fisher G, Ford JA, Poen JC, Vierra MA, Oberhelman H, et al. Preoperative chemoradiation for marginally resectable adenocarcinoma of the pancreas. J Gastrointest Surg. 2001;5(1):27-35.

11. Callery MP, Chang KJ, Fishman EK, Talamonti MS, William Traverso L, Linehan DC. Pretreatment assessment of resectable and borderline resectable pancreatic cancer: expert consensus statement. Ann Surg Oncol. 2009;16(7):1727-33.

12. Vauthey JN, Dixon E. AHPBA/SSO/SSAT Consensus Conference on Resectable and Borderline Resectable Pancreatic Cancer: rationale and overview of the conference. Ann Surg Oncol. 2009;16(7):1725-6.

13. National Comprehensive Cancer Network. Pancreatic Adenocarcinoma Version 2.2015. 2015. Accessed 31st Dec 2015

14. Varadhachary GR, Tamm EP, Abbruzzese $J$, Xiong HQ, Crane $\mathrm{CH}$, Wang $\mathrm{H}$, et al. Borderline resectable pancreatic cancer: definitions, management, and role of preoperative therapy. Ann Surg Oncol. 2006;13(8):1035-46.

15. Talamonti M. Borderline resectable pancreatic cancer: a new classification for an old challenge. Ann Surg Oncol. 2006;13(8):1019-20.

16. Heestand GM, Murphy JD, Lowy AM. Approach to patients with pancreatic cancer without detectable metastases. J Clin Oncol. 2015;33(16):1770-8.

17. Sauer R, Liersch T, Merkel S, Fietkau R, Hohenberger W, Hess C, et al. Preoperative versus postoperative chemoradiotherapy for locally advanced rectal cancer: results of the German CAO/ARO/AIO-94 randomized phase III trial after a median follow-up of 11 years. J Clin Oncol. 2012;30(16):1926-33.

18. van Hagen $P$, Hulshof MC, van Lanschot JJ, Steyerberg EW, van Berge Henegouwen MI, Wijnhoven BP, et al. Preoperative chemoradiotherapy for esophageal or junctional cancer. N Engl J Med. 2012;366(22):2074-84

19. Stessin AM, Meyer JE, Sherr DL. Neoadjuvant Radiation Is Associated With Improved Survival in Patients With Resectable Pancreatic Cancer: An Analysis of Data From the Surveillance, Epidemiology, and End Results (SEER) Registry. Int J Radiat Oncol Biol Phys. 2008;72(4):1128-33.

20. Moutardier V, Magnin V, Turrini O, Viret F, Hennekinne-Mucci S, Goncalves A et al. Assessment of pathologic response after preoperative chemoradiotherapy and surgery in pancreatic adenocarcinoma. Int J Radiat Oncol Biol Phys. 2004;60(2):437-43.

21. Gillen S, Schuster T, Meyer Zum Buschenfelde C, Friess H, Kleeff J. Preoperative/ neoadjuvant therapy in pancreatic cancer: a systematic review and metaanalysis of response and resection percentages. PLoS Med. 2010;7(4):e1000267.

22. Sasson AR, Wetherington RW, Hoffman JP, Ross EA, Cooper H, Meropol NJ, et al. Neoadjuvant chemoradiotherapy for adenocarcinoma of the pancreas: Analysis of histopathology and outcome. Int J Gastrointest Cancer. 2004, 34(2-3):121-7.

23. White RR, Xie HB, Gottfried MR, Czito BG, Hurwitz HI, Morse MA, et al. Significance of histological response to preoperative chemoradiotherapy for pancreatic cancer. Ann Surg Oncol. 2005:12(3):214-21.

24. Breslin TM, Hess KR, Harbison DB, Jean ME, Cleary KR, Dackiw AP, et al. Neoadjuvant chemoradiotherapy for adenocarcinoma of the pancreas: treatment variables and survival duration. Ann Surg Oncol. 2001;8(2):123-32.

25. Greer SE, Pipas JM, Sutton JE, Zaki BI, Tsapakos M, Colacchio TA, et al. Effect of neoadjuvant therapy on local recurrence after resection of pancreatic adenocarcinoma. J Am Coll Surg. 2008:206(3):451-7.

26. Koong AC, Le QT, Ho A, Fong B, Fisher G, Cho C, et al. Phase I study of stereotactic radiosurgery in patients with locally advanced pancreatic cancer. Int J Radiat Oncol Biol Phys. 2004;58(4):1017-21. 
27. Koong AC, Christofferson E, Le QT, Goodman KA, Ho A, Kuo T, et al. Phase II study to assess the efficacy of conventionally fractionated radiotherapy followed by a stereotactic radiosurgery boost in patients with locally advanced pancreatic cancer. Int J Radiat Oncol Biol Phys. 2005;63(2):320-3.

28. Schellenberg D, Goodman KA, Lee F, Chang S, Kuo T, Ford JM, et al. Gemcitabine chemotherapy and single-fraction stereotactic body radiotherapy for locally advanced pancreatic cancer. Int J Radiat Oncol Biol Phys. 2008;72(3):678-86.

29. Schellenberg D, Kim J, Christman-Skieller C, Chun CL, Columbo LA, Ford JM, et al. Single-fraction stereotactic body radiation therapy and sequential gemcitabine for the treatment of locally advanced pancreatic cancer. Int J Radiat Oncol Biol Phys. 2011;81(1):181-8.

30. Polistina F, Costantin G, Casamassima F, Francescon P, Guglielmi R, Panizzoni G, et al. Unresectable locally advanced pancreatic cancer: a multimodal treatment using neoadjuvant chemoradiotherapy (gemcitabine plus stereotactic radiosurgery) and subsequent surgical exploration. Ann Surg Oncol. 2010;17(8):2092-101.

31. Mahadevan A, Miksad R, Goldstein M, Sullivan R, Bullock A, Buchbinder E, et al. Induction gemcitabine and stereotactic body radiotherapy for locally advanced nonmetastatic pancreas cancer. Int J Radiat Oncol Biol Phys. 2011; 81(4):e615-22.

32. Lominska CE, Unger K, Nasr NM, Haddad N, Gagnon G. Stereotactic body radiation therapy for reirradiation of localized adenocarcinoma of the pancreas. Radiat Oncol. 2012;7(1):74

33. Tozzi A, Comito T, Alongi F, Navarria P, Iftode C, Mancosu P, et al. SBRT in unresectable advanced pancreatic cancer: preliminary results of a monoinstitutional experience. Radiat Oncol. 2013;8(1):148.

34. Gurka MK, Kim C, He AR, Charabaty A, Haddad N, Turocy J, et al. Stereotactic Body Radiation Therapy (SBRT) Combined With Chemotherapy for Unresected Pancreatic Adenocarcinoma. Am J Clin Oncol. 2014.

35. Herman JM, Chang DT, Goodman KA, Dholakia AS, Raman SP, Hacker-Prietz $A$, et al. Phase 2 multi-institutional trial evaluating gemcitabine and stereotactic body radiotherapy for patients with locally advanced unresectable pancreatic adenocarcinoma. Cancer. 2015;121(7):1128-37.

36. Katz MH, Pisters PW, Evans DB, Sun CC, Lee JE, Fleming JB, et al. Borderline resectable pancreatic cancer: the importance of this emerging stage of disease. J Am Coll Surg. 2008;206(5):833-46. discussion 846-838.

37. Skolnik JM, Barrett JS, Jayaraman B, Patel D, Adamson PC. Shortening the timeline of pediatric phase I trials: the rolling six design. J Clin Oncol. 2008; 26(2):190-5

38. Rajagopalan MS, Heron DE, Wegner RE, Zeh HJ, Bahary N, Krasinskas AM, et al. Pathologic response with neoadjuvant chemotherapy and stereotactic body radiotherapy for borderline resectable and locally-advanced pancreatic cancer. Radiat Oncol. 2013;8(1):254

39. Chuong MD, Springett GM, Freilich JM, Park CK, Weber JM, Mellon EA, et al. Stereotactic body radiation therapy for locally advanced and borderline resectable pancreatic cancer is effective and well tolerated. Int J Radiat Oncol Biol Phys. 2013;86(3):516-22.

40. Willett CG, Moughan J, O'Meara E, Galvin JM, Crane CH, Winter K, et al. Compliance with therapeutic guidelines in Radiation Therapy Oncology Group prospective gastrointestinal clinical trials. Radiother Oncol. 2012;105(1):9-13.

41. Dewas S, Bibault JE, Blanchard P, Vautravers-Dewas C, Pointreau Y, Denis F, et al. Delineation in thoracic oncology: a prospective study of the effect of training on contour variability and dosimetric consequences. Radiat Oncol. 2011;6(1):118

42. Khoo EL, Schick K, Plank AW, Poulsen M, Wong WW, Middleton M, et al. Prostate contouring variation: can it be fixed? Int J Radiat Oncol Biol Phys. 2012;82(5):1923-9.

43. Nijkamp J, de Haas-Kock DF, Beukema JC, Neelis KJ, Woutersen D, Ceha H et al. Target volume delineation variation in radiotherapy for early stage rectal cancer in the Netherlands. Radiother Oncol. 2012;102(1):14-21.

\section{Submit your next manuscript to BioMed Central and we will help you at every step:}

- We accept pre-submission inquiries

- Our selector tool helps you to find the most relevant journal

- We provide round the clock customer support

- Convenient online submission

- Thorough peer review

- Inclusion in PubMed and all major indexing services

- Maximum visibility for your research

Submit your manuscript at www.biomedcentral.com/submit
Biomed Central 